\title{
Communication \\ PIV Measurements of Turbulent Airflow in a Channel with Trapezoidal Ribs on One Wall
}

\author{
Waldemar Wodziak * and Jacek Sobczyk (D)
}

Strata Mechanics Research Institute, Polish Academy of Sciences, 30-059 Krakow, Poland; sobczyk@imgpan.pl

* Correspondence: wodziak@imgpan.pl

Citation: Wodziak, W.; Sobczyk, J. PIV Measurements of Turbulent

Airflow in a Channel with

Trapezoidal Ribs on One Wall. Appl.

Sci. 2022, 12, 1581. https://doi.org/

10.3390/app12031581

Academic Editor: Cesare Biserni

Received: 22 December 2021

Accepted: 27 January 2022

Published: 1 February 2022

Publisher's Note: MDPI stays neutral with regard to jurisdictional claims in published maps and institutional affiliations.

Copyright: () 2022 by the authors. Licensee MDPI, Basel, Switzerland. This article is an open access article distributed under the terms and conditions of the Creative Commons Attribution (CC BY) license (https:/ / creativecommons.org/licenses/by/ $4.0 /)$.

\begin{abstract}
This paper presents the results of PIV measurements of turbulent airflow in a channel with a single ribbed wall. The rough elements had the form of evenly spaced trapezoids. The ratio of the height of a single rib to the height of the measurement channel was 0.1 . The ratio of the distance between adjacent ribs to the height of a single rib (i.e., the $\mathrm{w} / \mathrm{k}$ ratio) was 5 , which suggests a transitional region within the airflow structure between the $\mathrm{d}$ - and k- types. The measurements were carried out for the inlet velocity, at which the Reynolds number (Re) was equal to 32,500. The distance to the first rib, at which the turbulent velocity profile is fully developed, was determined. The distribution of the average velocity components, the velocity fluctuation components, turbulent shear stress, as well as the turbulence kinetic energy between two adjacent ribs was presented. The analysis of average streamlines indicated the existence of two vortex structures between the ribs.
\end{abstract}

Keywords: turbulent flow; trapezoidal ribs; PIV; surface roughness

\section{Introduction}

Turbulent flows in a ribbed channel are present in numerous fields of engineering, such as heat exchange [1,2] turbines [3], maritime engineering [4], and the mining industry [5]. The structure of the airflow between two adjacent ribs differs depending on the Reynolds number and the distance between the ribs. Perry et al. [6] suggested that the flow between ribs should be divided into a d-type and a k-type flow. The former applies to a geometrical configuration, where the distance between two adjacent ribs is small. In this case, stationary vortex is present in the cavity between the two ribs. The mass transfer between the cavity and the airflow over the rib is minor. When the distance between two adjacent ribs is greater than a certain limit value, the stream on the leeward slope of the rib becomes detached. When the $\mathrm{w} / \mathrm{k}$ ratio (where " $\mathrm{w}$ " is the distance between the ribs and " $\mathrm{k}$ " is the rib height) reaches a certain limit value, the stream becomes reattached between the ribs and a recirculation zone arises downstream of the leeward slope of the rib.

Coleman et al. [7] presented studies of turbulent flow in a channel with repeated roughness for the $\mathrm{w} / \mathrm{k}$ ratio ranging from 1 to 16 . The transition from the d-type flow to the k-type flow occurs at $w / k=5$. A transitional state is present at that point. Stable vortices occur between the ribs in the case of the d-type flow. In the case of the k-type flow, two vortices characterized by an opposite rotation direction are present between the ribs. Numerical calculations based on the large eddy simulation (LES) method, confirming the presence of both k- and d-type flows, were carried out by Cui et.al [8]. The boundary between a change in the flow structure occurs for the $\mathrm{w} / \mathrm{k}$ ratio equal to 4 . In the same publication, it was also confirmed that the flow structure also depends on the height of a single rib (k). In [9] the authors presented the results of studies, conducted using the particle image velocimetry (PIV) method, of water flow in a channel with rough, squareshaped ribs within the 1400-50,000 Re number range. The ratio of the height of a single rough element to the distance between such elements was $1 / 10$ and $1 / 20$. The authors concluded that vortices were forming near the top edge of the rib. They also noticed such 
phenomena as recirculation, detachment, and reattachment of the stream, the range of which only depended on the Re number to a minor extent. Wang et al. [10] performed PIV measurements in a ribbed channel for $\operatorname{Re}=22,000$. The flow structure near the rib was identified. A probability density function (PDF) analysis of velocity fluctuation was carried out, which demonstrated the existence of two modes in the shearing layer. Additionally, the vortex topology was determined. Leonardi et al. [11] performed numerical calculations using the direct numerical simulation (DNS) method in a channel with a single ribbed wall (with square-shaped ribs). Simulations were conducted for the conditions of a fully developed turbulent flow for the 8800-20,800 Re number range. Analyses were carried out for $\operatorname{Re}=8800$. The authors analyzed the $\mathrm{w} / \mathrm{k}$ ratio in several configurations from 0.33 to 19 . Two vortices with the opposite rotation direction appeared in the cavity between the ribs for the $\mathrm{w} / \mathrm{k}$ ratio below 4 . When the $\mathrm{w} / \mathrm{k}$ ratio was equal to or greater than 7 , the flow was reattached on the bottom wall.

The problem of turbulent flow near square rough elements was examined by Stoesser et al. in [12]. The authors performed numerical calculations using the LES method for two flow regimes: of the k-type $(\mathrm{w} / \mathrm{k}=9)$ and a transitional one between the $\mathrm{d}$ - and the k-type $(\mathrm{w} / \mathrm{k}=4.5)$. They observed differences in the flow structure between the two regimes despite the fact that in both regimes water flowed into and out of the cavity between the ribs, and despite the fact that there was a recirculation zone present in the cavity. The calculations performed using the LES method yielded similar results to the measurements carried out by Coleman et al. [7] as well as the numerical calculations performed using the DNS method by Leonardi et al. [11].

Square-shaped ribs are the most common rough elements described in the literature. However, the problem of flow in a channel with a trapezoidal rib has only been described in a few studies. Using the PIV and liquid crystal thermography (LCT) methods, Ali et al. [13] determined the effect the acute angle of the right-angled trapezoid on the flow structure and the distribution of heat downstream the rib for four Reynolds numbers within the 9400-61,480 range. For $\operatorname{Re}=9400$, the range of the recirculation zone is independent of the angle. For the remaining Re numbers, the recirculation zone increases in size with the increase in the angle of the trapezoid. In their publication, Skotniczny and Ostrogórski [5] presented the results of measurements and numerical simulations of an airflow near a side wall of a mining longwall working with supports, the elements of which resembled trapezoidal ribs. Velocity measurement results collected in an actual mine were compared with the results of a two-dimensional CFD simulation carried out using a k- $\omega$ scale adaptive simulation SAS turbulence model. The velocity value determined experimentally was consistent, to a satisfactory degree, with the velocity obtained through calculations. Large discrepancies between the measurements and the simulation were obtained for turbulence intensity as well as for the kinetic energy of the turbulence.

The results of the measurements presented in this paper provide additional information on the problem of transitional flow occurring between two rough elements and can be used for the purpose of comparison with numerical calculations.

\section{Materials and Methods}

The experimental setup used for flow testing in a channel with one rough wall consisted of a single radial fan, a section designed to equalize the flow and a measuring channel. Due to its size, the experimental setup is presented in two figures: Figures 1 and 2. The radial fan, with inverter-controlled rotational speed, supplied air into the flow equalization section consisting of the following elements: a straightener, a diffuser with two rows of grids, and the first and second confusor connected with the measuring channel (Figure 1). 


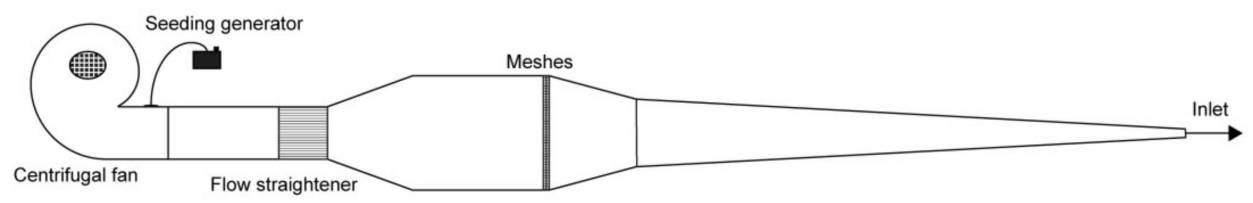

Figure 1. Schematic view of the test station-flow straightening elements.

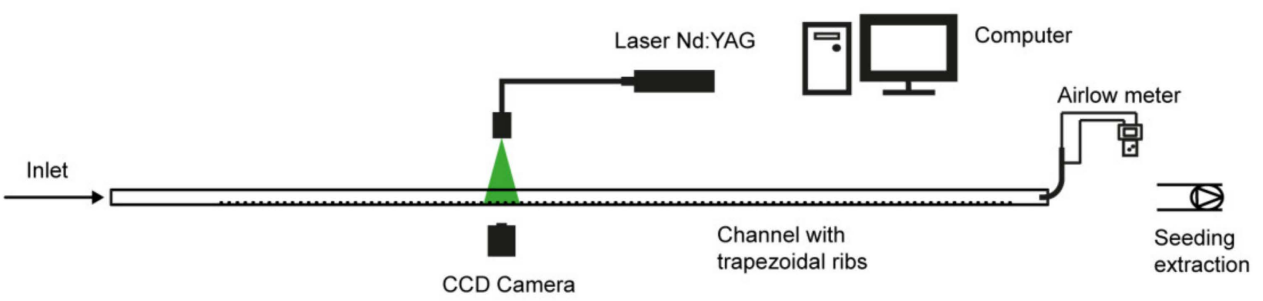

Figure 2. Schematic view of the experimental setup—the channel featuring measuring instruments.

The $1.2 \mathrm{~m}$ long measuring channel with a $0.20 \mathrm{~m} \times 0.02 \mathrm{~m}$ internal cross-section was made of mineral glass. The top wall and the side walls were smooth along their entire length, whereas the bottom wall was smooth only along its initial $(0.14 \mathrm{~m})$ and its final $(0.1 \mathrm{~m})$ sections. Along its remaining length, the bottom wall featured periodic roughness in the form of evenly spaced, isosceles trapezoid shaped ribs positioned crosswise in relation to the direction of the flow. The ribs represented a simplified laboratory model of ribbed, arched supports used in mining longwall workings. The dimensions of the ribs (in millimeters) are presented in Figure 3.

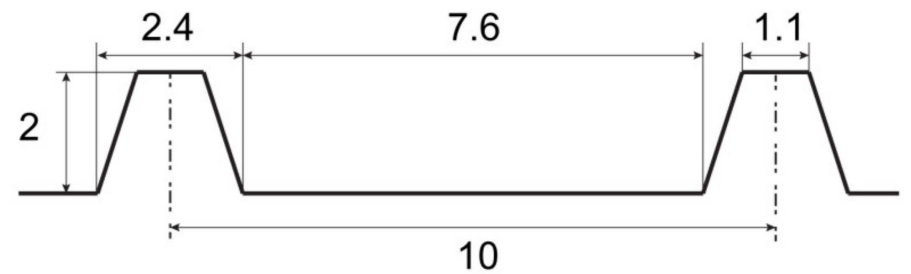

Figure 3. Trapezoidal ribs (dimensions in $\mathrm{mm}$ ).

Velocity field measurements were carried out using the PIV method. The PIVTEC GmbH Goettingen, Germany aerosol generator with Laskin atomizer nozzles supplied DiEthyl-Hexyl-Sebacat (DEHS) marker particles into the channel through the suction opening of the fan. Based on the air dynamic viscosity $18.13 \mathrm{~Pa} \cdot \mathrm{s}$, DEHS particle diameter $1 \mu \mathrm{m}$ and density $912 \mathrm{~kg} / \mathrm{m}^{3}$, channel height $0.02 \mathrm{~m}$ and mean velocity $29 \mathrm{~m} / \mathrm{s}$ Stokes number is about 0.004 . For Stokes number smaller than one particle, fluid streamlines were followed closely. The tested area was illuminated with a light sheet generated using a neodymium laser. An scientific Complementary Metal-Oxide-Semiconductor (sCMOS) camera, with its optical axis oriented perpendicularly to the plane of the laser sheet, recorded two pictures in a short interval. Each measurement consisted of a series of double pictures captured at a frequency of $15 \mathrm{~Hz}$. The pictures were then sent to a PC, where dedicated software calculated instantaneous velocity fields, which were subsequently averaged over time.

Numerous initial tests were carried out prior to conducting the actual measurements. Optimal measuring conditions were determined on the basis of these tests and defects of the test station, e.g., a local leak of the channel, were identified and removed. It was ascertained that the length of the test station was sufficient for obtaining the required development of the velocity profile. During each of the measurements, the air velocity in the channel was controlled with a Pitot tube installed at the outlet and connected to a TSI Incorporated, Minnesota, USA Airflow meter. The Reynolds number, based on height 
$\mathrm{H}=0.02 \mathrm{~m}$ and the average velocity in the initial, i.e., smooth section of the channel, was $\operatorname{Re}=32,500$.

\section{Results and Discussion}

\subsection{Development of Turbulent Flow}

After measuring the velocity at the channel inlet, experiments were conducted to determine the distance from the inlet, at which turbulent flow was fully developed. Turbulent flows are considered fully developed if the velocity profile does not change along the flow axis. The first stage of experimental studies included velocity measurements at $\mathrm{x} / \mathrm{H}$ distance ranging from 18.6 to 49.6 from the inlet. A total of 332 double pictures were recorded, which were further analyzed using the adaptive correlation method. The instantaneous vector velocity fields obtained in this manner were averaged over time. The velocity was normalized by the average velocity over the rib $U_{r}=29 \mathrm{~m} / \mathrm{s}$. Figure 4 presents the $\mathrm{U} / \mathrm{U}_{\mathrm{r}}$ velocity distribution at the $\mathrm{x} / \mathrm{H}=18.6, \mathrm{x} / \mathrm{H}=20.6, \mathrm{x} / \mathrm{H}=27.6, \mathrm{x} / \mathrm{H}=41.6$ and $\mathrm{x} / \mathrm{H}=49.6$ distances from the first rib.

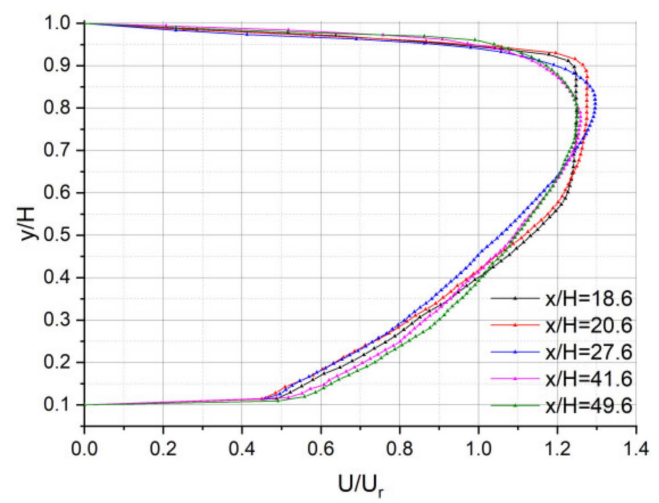

(a)

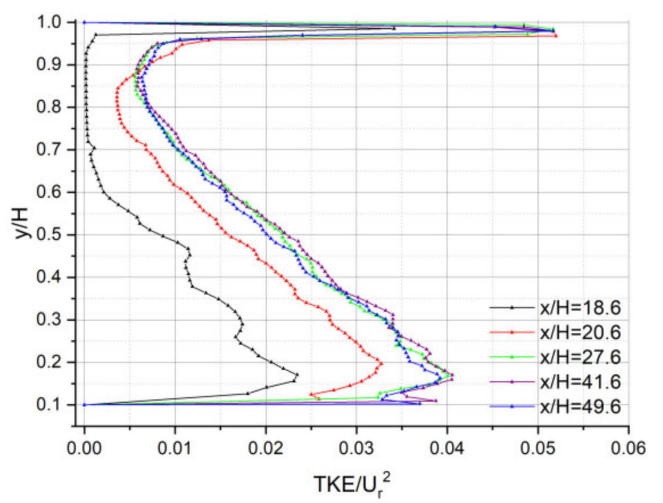

(b)

Figure 4. Distribution of the normalized streamwise velocity component: (a) and turbulence kinetic energy; (b) at distances $\mathrm{x} / \mathrm{H}=18.6, \mathrm{x} / \mathrm{H}=20.6, \mathrm{x} / \mathrm{H}=27.6, \mathrm{x} / \mathrm{H}=41.6$ and $\mathrm{x} / \mathrm{H}=49.6$ from the first rib.

At $y / H$ height ranging from 0.6 to 0.9 , the profile of the streamwise velocity component obtained at distances $x / H=18.6$ and $x / H=20.6$ is almost flat. The profile becomes more convex with increasing distance from the inlet. The profiles at distances of $x / H=27.6$ and 41.6 matched each other. This meant that turbulent flow was fully developed for $\mathrm{x} / \mathrm{H}$ between 27.6 and 41.6. The profile measured at $x / H=49.6$ differed from the remaining profiles, as it was close to the outlet of the measuring channel. The profiles of normalized turbulence kinetic energy (TKE) were consistent at x/H 27.6 and 41.6.

\subsection{Average Values of Velocity Components}

The presented below average values of the velocity field are the result of averaging out 1200 instantaneous velocity fields. The spatial scale was divided by the height of the rib $\mathrm{k}=0.002 \mathrm{~m}$ and the calculated velocity values by the average velocity over the rib $\mathrm{U}_{\mathrm{r}}=29 \mathrm{~m} / \mathrm{s}$. The results of the measurements were presented between two adjacent ribs within the height range from 0 to $4 \mathrm{k}$.

Figure 5 presents averaged streamlines. The flow detaching along the top edge of the rib forms a zone consisting of two minor vortices with identical rotation direction in the cavity. The center of the vortex is located at $\mathrm{x} / \mathrm{k}=1.9, \mathrm{y} / \mathrm{k}=0.6$ distance at the windward $\mathrm{rib}$ and at $\mathrm{x} / \mathrm{k}=3.9, \mathrm{y} / \mathrm{k}=0.4$ distance at the leeward rib. 


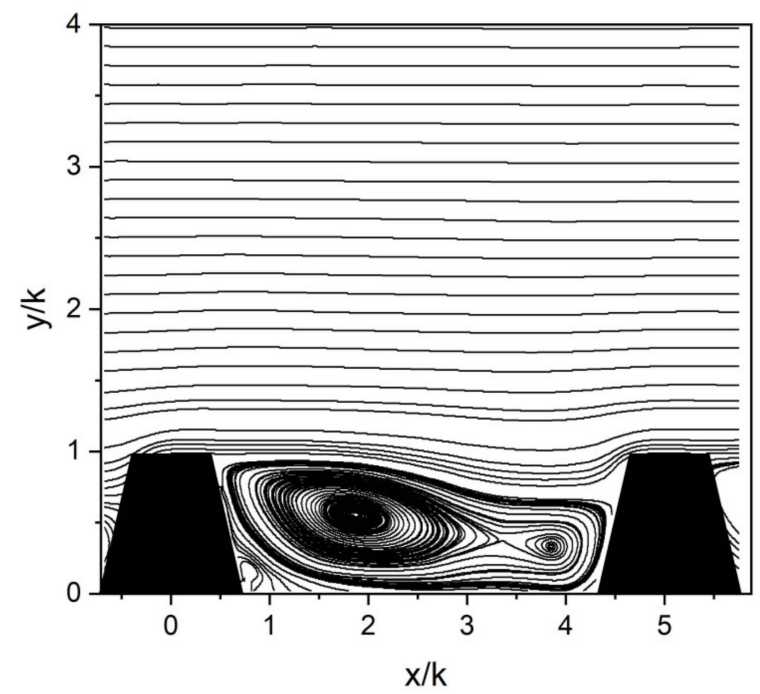

Figure 5. Average streamlines in the cavity.

Figure 6 presents scalar maps and profiles of streamwise $U$ and transverse $V$ velocity components at $\mathrm{x} / \mathrm{k}$ distances equal to $0,1,2.5,4$ and 5 . The profiles of the U velocity component obtained over the ribs at $x / k=0$ and $x / k=5$ are almost identical. Due to the presence of vortices in the cavity, the $U$ component between the ribs has a negative value for $\mathrm{x} / \mathrm{k}=1$ at the height $\mathrm{y} / \mathrm{k}=0.7$, for $\mathrm{x} / \mathrm{k}=2.5$ at the height $\mathrm{y} / \mathrm{k}=0.5$ and for $\mathrm{x} / \mathrm{k}=4$ at the height $\mathrm{y} / \mathrm{k}=0.35$. The presence of the recirculation zone is also visible in the distribution of the $\mathrm{V}$ transverse component. A shearing layer and local acceleration occur over the rib.
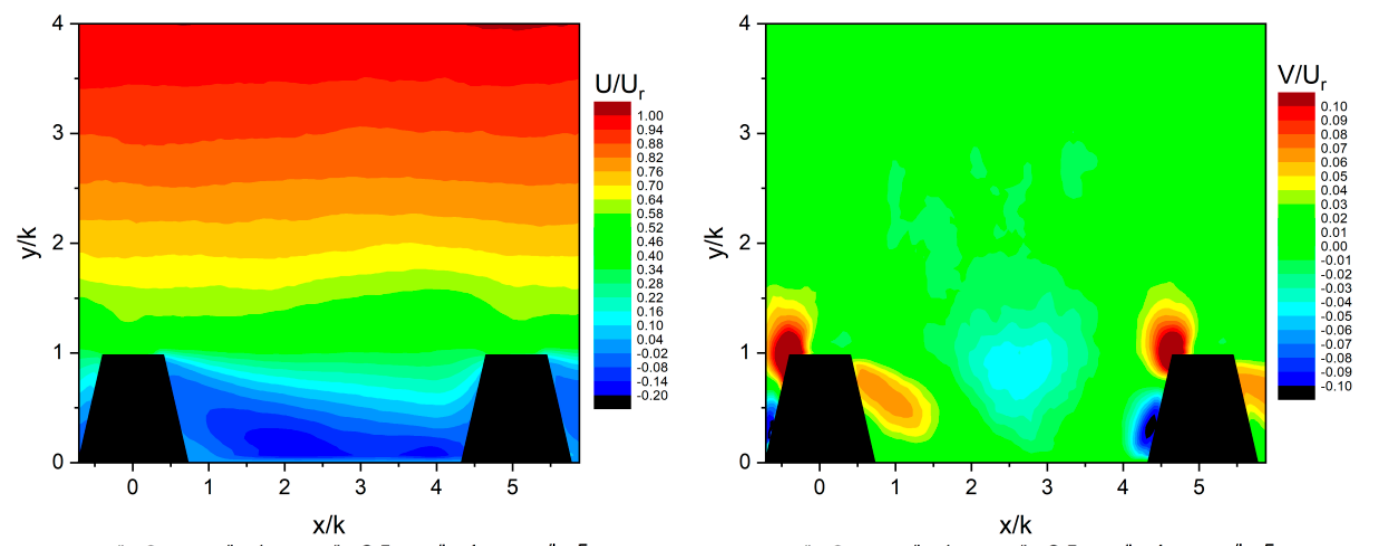

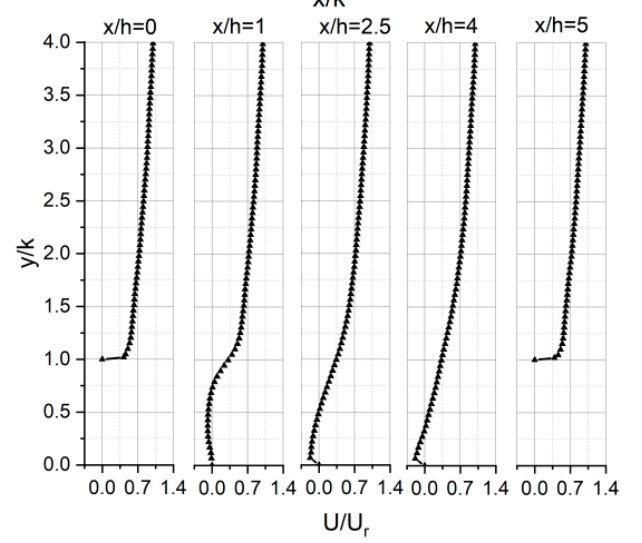

(a)

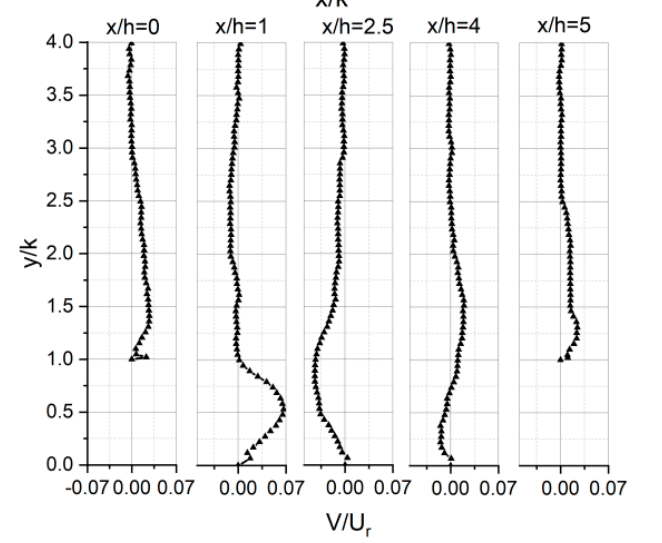

(b)

Figure 6. Average normalized U (a); and V (b) velocity components. 
Figures 7 and 8 present statistical values that accompany the turbulence, such as Reynolds stress and turbulence kinetic energy. The results in the direct vicinity of the edge carry a significant uncertainty. Figure 7 presents rms (root mean square) velocity values $\left(U_{r m s}\right.$ and $\left.V_{r m s}\right)$. Except at the top of the ribs, the maximum $U_{r m s}$ values occurred near the middle of the distance between the ribs at the height $\mathrm{y} / \mathrm{k}=1.2$. Similar results were obtained through simulations by Cui et al. [8] with the maximum $U_{\text {rms }}$ value identified closer to the leeward rib. The maximum $V_{\text {rms }}$ values were obtained for $x / k$ equal to 3.2 at the height $y / k=0.75$. The results of $U_{\text {rms }}$ and $V_{\text {rms }}$ components are qualitatively consistent with the results obtained by Stoesser et al. [12].
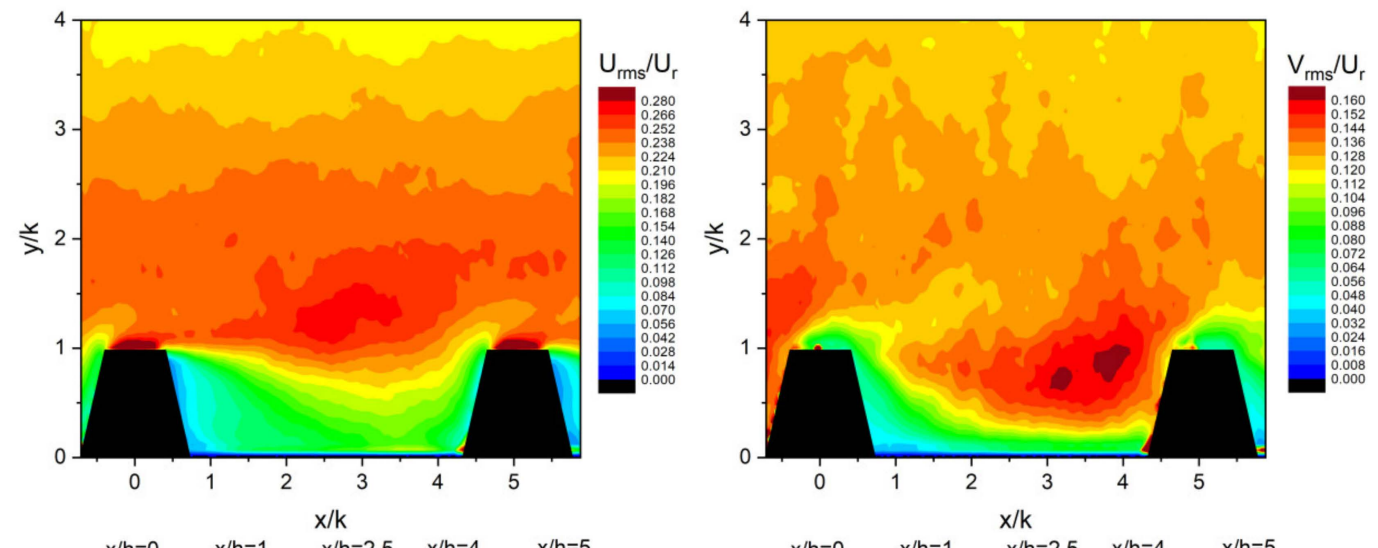

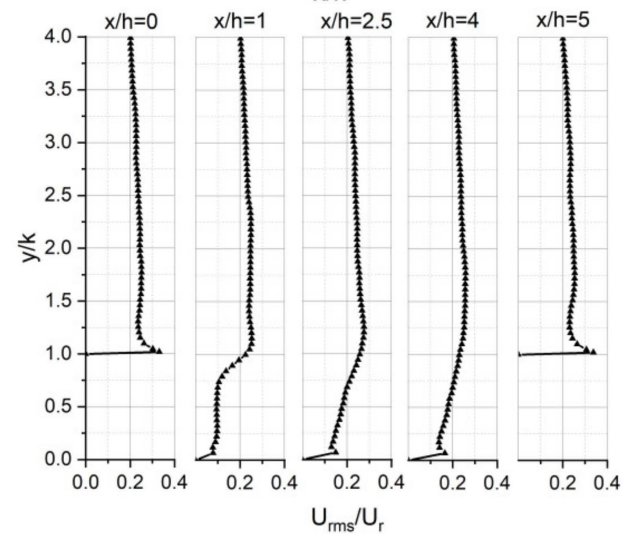

(a)

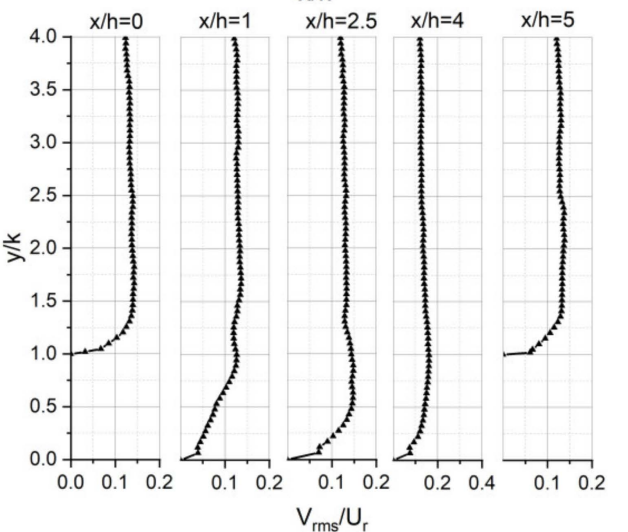

(b)

Figure 7. Average normalized $\mathrm{U}_{\mathrm{rms}}(\mathbf{a})$; and $\mathrm{V}_{\mathrm{rms}}(\mathbf{b})$ velocity components.

Figure 8 presents average, normalized, turbulent shear stress $-\mathrm{u}^{\prime} \mathrm{v}^{\prime}$ and the turbulence kinetic energy (TKE). The shearing layer forming over the rough elements is clearly visible (the red area) on the outline of the $-u^{\prime} v^{\prime}$ shear stress. The maximum values of turbulent shear stress were obtained at the $\mathrm{x} / \mathrm{k}$ distance equal to 3.5. The zone of high values of turbulence kinetic energy overlaps to a large extent with high values of turbulent shear stress. Mass and momentum transfer occurs between the cavity and the zone present over the roughness, with the highest intensity for $\mathrm{x} / \mathrm{k}$ ranging from 2 to 4 . 

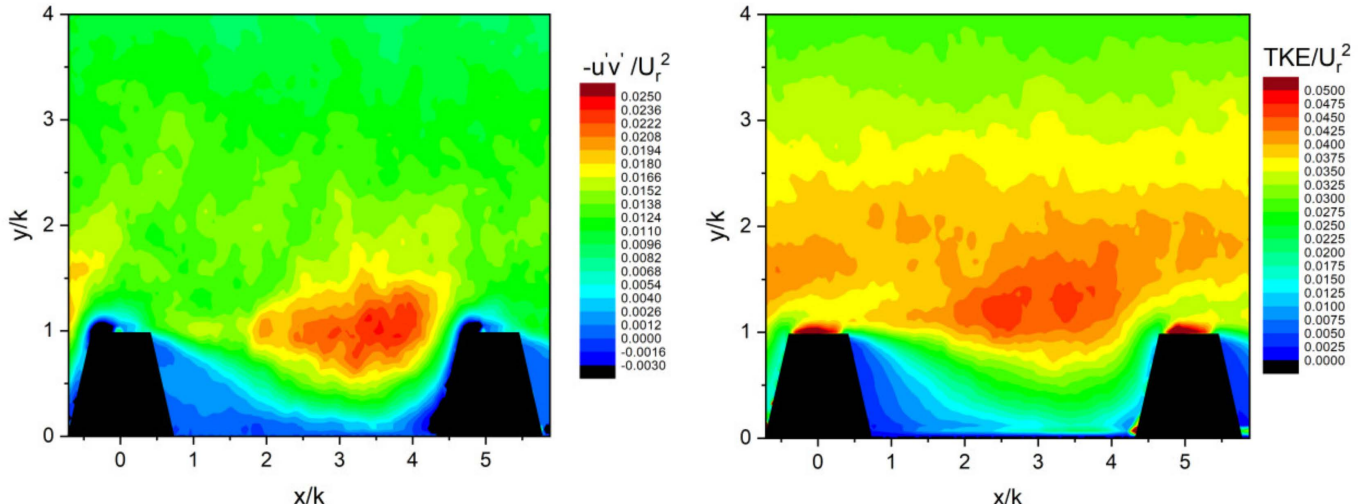

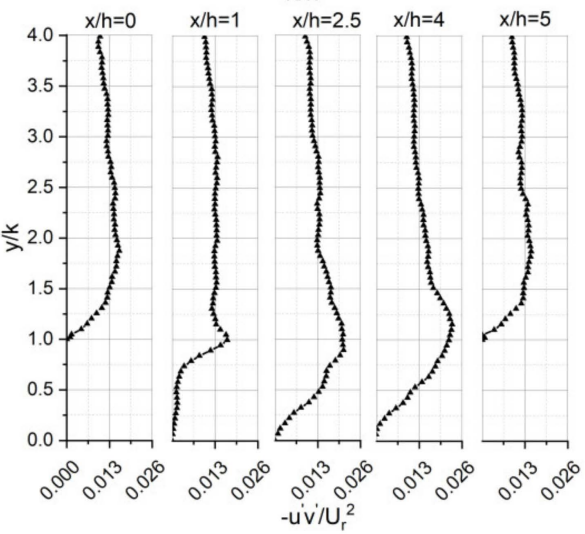

(a)

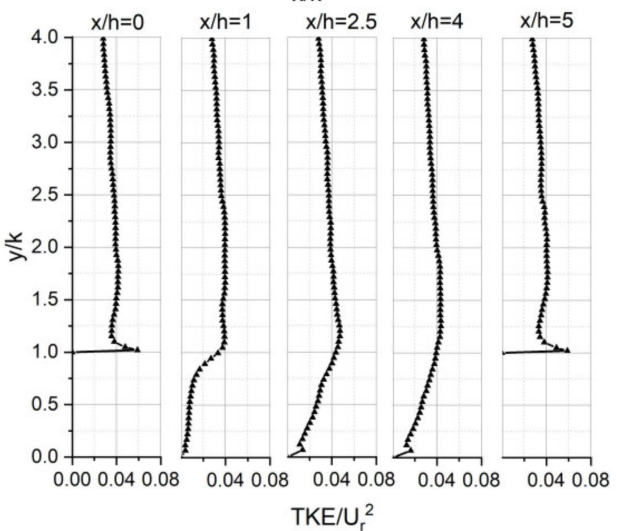

(b)

Figure 8. Averaged, normalized Reynolds shear stress values $-u^{\prime} v^{\prime}(a)$; and turbulence kinetic energy (b) values.

\subsection{Instantaneous Velocity Field}

Vortices are generated in the shearing layer as the stream detaches from the top edge of the rib. Galileo's decomposition is one of the methods of the visualization of non-stationary vortices [14]. The total velocity $U$ can be expressed as sum of convection velocity $U_{c}$ and deviation $\mathrm{u}_{\mathrm{c}}$. Galilean transformation removes a constant that is independent in space and time. When convection velocity matches vortices velocity, it becomes visible as a circular pattern of velocity vectors. Two reference velocities were removed from velocity vector field equal to 0.5 and 0.86 of the $U_{\mathrm{r}}$ velocity, respectively. There are several vortices coming off of the windward rib visible in Figure 9a. The visualization shows that multi-scale vortices are present in the shearing layer. These have the size of approx. 1/3-1/2 of the rib height. The angle between the path of the vortices and the $x$ axis is ca. $25^{\circ}$. Figure $9 \mathrm{~b}$ shows three vortices between the ribs, of the same size as the vortices appearing in the shearing layer. 


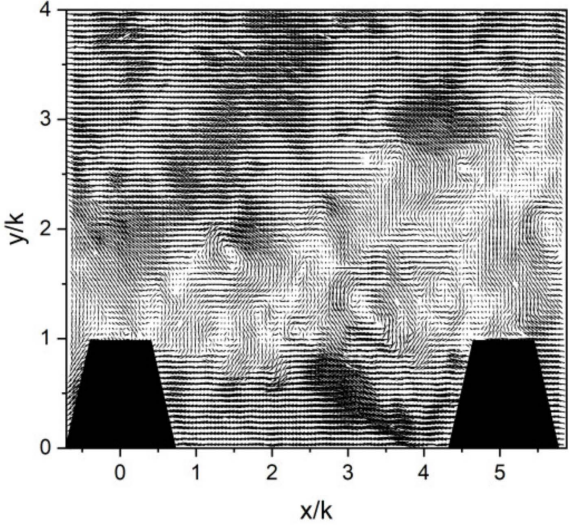

(a)

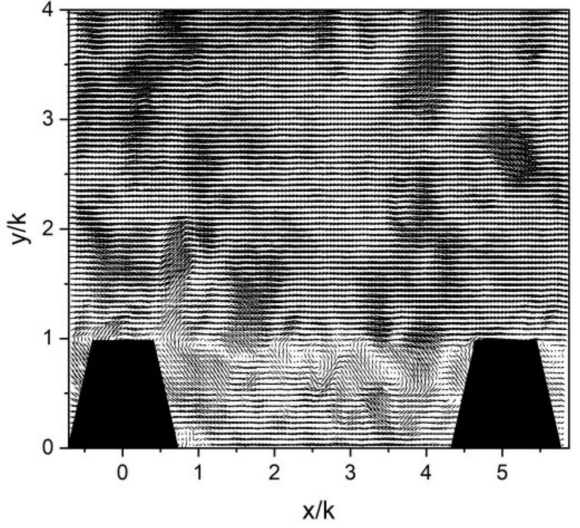

(b)

Figure 9. Instantaneous velocity fields for the reference velocity equal to $0.5 \mathrm{U}_{\mathrm{r}}(\mathbf{a})$; and $0.86 \mathrm{U}_{\mathrm{r}}(\mathbf{b})$.

\section{Conclusions}

The paper presents results for turbulent flow between trapezoidal ribs. In the case of the tested topology (with $\mathrm{w} / \mathrm{k}=5$ ), the flow was characterized by a transitional state between the $\mathrm{d}$ - and the k-type. Intense mass and momentum transfer occurred between the cavity located between the ribs and the remaining part of the channel. Two vortices characterized by identical rotation direction were formed in the recirculation zone between the ribs. The observed flow structures are consistent with those described in the literature for the transitional regime. The collected database can be used for the purpose of comparisons with numerical calculations.

Author Contributions: Conceptualization, W.W. and J.S.; methodology, W.W.; validation, W.W. and J.S.; formal analysis, W.W. and J.S.; investigation, W.W. and J.S.; writing-original draft preparation, W.W.; writing-review and editing, W.W. and J.S.; visualization, W.W.; supervision, W.W. All authors have read and agreed to the published version of the manuscript.

Funding: This research was completed in 2021, as part of a statutory work carried out at the Strata Mechanics Research Institute of the Polish Academy of Sciences in Krakow (Poland), funded by the Ministry of Science and Higher Education.

Institutional Review Board Statement: Not applicable.

Informed Consent Statement: Not applicable.

Data Availability Statement: The data presented in this study are available upon request from the corresponding author.

Acknowledgments: The authors express their gratitude to Jerzy Krawczyk and Przemysław Skotniczny for the valuable guidance and help in preparatory work.

Conflicts of Interest: The authors declare no conflict of interests.

\section{References}

1. Viswanathan, A.K.; Tafti, D.K. Detached Eddy Simulation of turbulent flow and heat transfer in a ribbed duct. ASME J. Fluids Eng. 2005, 127, 888-896. [CrossRef]

2. Ruck, S.; Arbeiter, F. Detached eddy simulation of turbulent flow and heat transfer in cooling channels roughened by variously shaped ribs on one wall. Int. J. Heat Mass Transf. 2018, 118, 388-401. [CrossRef]

3. Bons, J.P. A review of surface roughness effects in gas turbines. J. Turbomach. 2010, 132, 021004. [CrossRef]

4. Schultz, M.P. Effects of coating roughness and biofouling on ship resistance and powering. Biofouling 2007, 23, 331-341. [CrossRef] [PubMed]

5. Skotniczny, P.; Ostrogórski, P. Three-dimensional air velocity distributions in the vicinity of a mine heading's sidewall. Arch. Min. Sci. 2018, 63. [CrossRef]

6. Perry, A.E.; Schofield, W.H.; Joubert, P.N. Rough wall turbulent boundary layers. J. Fluid Mech. 1969, 37, 383-413. [CrossRef] 
7. Coleman, S.E.; Nikora, V.I.; Mclean, S.R.; Schlicke, E. Spatially Averaged Turbulent Flow over Square Ribs. J. Eng. Mech. 2007, 133, 194-204. [CrossRef]

8. Cui, J.; Patel, V.C.; Lin, C.L. Large-eddy simulation of turbulent flow in a channel with rib roughness. Int. J. Heat Fluid Flow 2003, 24, 372-388. [CrossRef]

9. Islam, S.M.; Haga, K.; Kaminaga, M.; Hino, R.; Monde, M. Experimental analysis of turbulent flow structure in a fully developed rib-roughned rectangular channel with PIV. Exp. Fluids 2002, 33, 296-306. [CrossRef]

10. Wang, L.; Salewski, M.; Sundén, B. Turbulent flow in a ribbed channel: Flow structures in the vicinity of a rib. Exp. Therm. Fluid Sci. 2010, 34, 165-176. [CrossRef]

11. Leonardi, S.; Orlandi, P.; Smalley, R.J.; Djenidi, L.; Antonia, R.A. Direct numerical simulations of turbulent channel flow with transverse square bars on one wall. J. Fluid Mech. 2003, 491, 229-238. [CrossRef]

12. Stoesser, T.; Nikora, V.I. Flow structure over square bars at intermediate submergence: Large Eddy Simulation study of bar spacing effect. Acta Geophys. 2008, 56, 876-893. [CrossRef]

13. Ali, M.S.; Tariq, A.; Gandhi, B.K. Flow and heat transfer investigation behind trapezoidal rib using PIV and LCT measurements. Exp. Fluids 2013, 54,1-15. [CrossRef]

14. Adrian, R.J.; Christensen, K.T.; Liu, Z.C. Analysis and interpretation of instantaneous turbulent velocity fields. Exp. Fluids 2000, 29, 275-290. [CrossRef] 